\title{
The Potts model built on sand
}

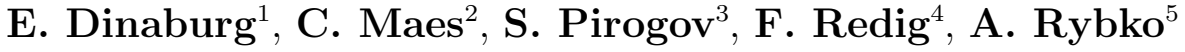

\begin{abstract}
We consider the $q=4$ Potts model on the square lattice with an additional hard-core nonlocal interaction. That interaction arises from the choice of the reference measure taken to be the uniform measure on the recurrent configurations for the abelian sandpile model. In that reference measure some correlation functions have a power-law decay. We investigate the low-temperature phase diagram and we prove the existence of a single stable phase with exponential decay of correlations. For all boundary conditions the density of 4 in the infinite volume limit goes to one as the temperature tends to zero.
\end{abstract}

\section{MODEL}

We define the model on the two-dimensional lattice $\boldsymbol{Z}^{2}$. Lattice sites are denoted by $x, y, z$ and we write $x \sim y$ if $x$ and $y$ are nearest neighbors. For a subset $V \subset Z^{2}$ we denote by $\partial V$ the exterior boundary of $V$, i.e., the set of those $y \in Z^{2} \backslash V$ such that there exists a nearest neighbor of $y$ in $V, \bar{V}=V \cup \partial V$, and the set $\partial\left(\boldsymbol{Z}^{2} \backslash V\right)$ is called the inner boundary of $V$. The square $[-n, n]^{2} \cap \boldsymbol{Z}^{2}$ is denoted by $V_{n}$. The configuration space is $\Omega=\{1,2,3,4\}^{\boldsymbol{Z}^{2}}$. Elements of $\Omega$ are written as $\eta, \xi$. For a configuration $\eta, \eta(x) \in\{1,2,3,4\}$ is interpreted as the "number of sand grains" at $x$. For $n \in \mathbb{N}, \Omega_{n}=\{1,2,3,4\}^{V_{n}}$ denotes the set

\footnotetext{
${ }^{1}$ Institute of Physics of Earth, RAS, Moscow

${ }^{2}$ Instituut voor Theoretische Fysica, K.U.Leuven email: christian.maes@fys.kuleuven.ac.be

${ }^{3}$ Institute for Information Transmission Problems, RAS, Moscow, supported by RFFI-grant 02-01-01276

${ }^{4}$ Faculteit Wiskunde en Informatica, Technische Universiteit Eindhoven

${ }^{5}$ Institute for Information Transmission Problems, RAS, Moscow, supported by RFFI-grant 02-01-00068
} 
of finite volume height configurations. Below we introduce measures $\mu_{\beta, n}^{a}$ on $\Omega_{n}$ that correspond to the finite volume Potts model at inverse temperature $\beta$ with boundary condition $a \in\{1,2,3,4\}$, restricted to a special set of "recurrent configurations" defined from the abelian sandpile model (cf. subsection 1.2 below). Our main result is that for $\beta$ large, $\mu_{\beta, n}^{4}$ forms the single stable phase of that model.

1.1. Potts model. The Potts Hamiltonian with fixed boundary condition $a \in\{1,2,3,4\}$ on the volume $V_{n}$ is

$$
H_{n}(\eta \mid a)=\sum_{x \sim y \in V_{n}} I[\eta(x) \neq \eta(y)]
$$

That is a finite sum over nearest neighbor pairs of sites of which at least one belongs to $V_{n}$ and where it is understood that we substitute $\eta(z)=a$ whenever $z \notin V_{n}$.

The Hamiltonians $H_{n}(\eta \mid a)$ give rise to the finite volume Gibbs measures on $\Omega_{n}$ :

$$
\nu_{\beta, n}^{a}(\eta)=\frac{1}{Z_{\beta, n}^{a}} \exp \left[-\beta H_{n}(\eta \mid a)\right]
$$

where $\beta>0$ is the inverse temperature and where the normalizing factor $Z_{\beta, n}^{a}$ is the partition function. It is well-known that there exists a critical inverse temperature $\beta_{c} \in(0,+\infty)$ such that for $\beta<\beta_{c}$ the Potts model has a unique infinite volume Gibbs measure (as $n \uparrow+\infty$ ), i.e., the weak limits of $\nu_{\beta, n}^{a}$ for different $a$ coincide, while for $\beta<\beta_{c}$, 
these weak limits are all different and define 4 mutually singular ergodic Gibbs measures $\nu_{\beta}^{a}$ on $\Omega$, called the pure phases.

1.2. Sandpile model. The abelian sandpile model in volume $V_{n}$ is a Markov chain on $\Omega_{n}$. We briefly introduce that Markov chain, more details can be found in the original paper [1], and in [2] or [9].

The Markov chain starting from $\eta_{0} \in \Omega_{n}$ is defined as follows. Suppose that $\eta_{t-1}$ is the configuration at time $t-1 \geq 0$. Pick randomly a site in $V_{n}$, say $x \in V_{n}$, and add one grain at $x$ to $\eta_{t-1}$. In case $\eta_{t-1}(x) \leq 3$, the new configuration is simply

$$
\eta_{t}(y)=\eta_{t-1}(y)+\delta_{x, y}
$$

with $\delta_{x, y}$ the Kronecker delta. In case $\eta_{t-1}(x)=4$, by adding one grain at $x$ the number of grains at $x$ becomes equal to 5 . That site will now topple, i.e., 4 grains are removed from $x$ and one grain is given to each neighbor of $x$ in $V_{n}$. At the boundary, grains are lost when the site topples. It is now possible that the number of grains at one or more neighbors of $x$ exceeds 4 and we have to repeat the toppling operation on all of these, and so on. It turns out that no matter in what order we perform these toppling operations, at the end of the avalanche a unique configuration $\eta_{t} \in \Omega_{n}$ appears. In that way, a discrete time Markov chain on $\Omega_{n}$ is defined where the only randomness 
is in the independently repeated uniform choice of the site where a grain is added.

Analysis of that Markov chain learns that it has a unique class $\mathcal{R}_{n}$ of recurrent configurations and the stationary measure $\lambda_{n}$ is uniform on that class:

$$
\lambda_{n}(\eta)=\frac{1}{\left|\mathcal{R}_{n}\right|} I\left[\eta \in \mathcal{R}_{n}\right]
$$

see [2].

Whether a particular configuration $\eta \in \Omega_{n}$ belongs to $\mathcal{R}_{n}$ can be decided from the output of the so-called burning algorithm [2]. The burning algorithm has as an input the configuration $\eta$ and its output is a set $A \subset V_{n}$. It runs as follows: start from $A_{0}=V_{n}$ and remove ("burn") all those vertices $x \in A_{0}$ (and edges containing $x$ ) which satisfy $\eta(x)>n_{A_{0}}(x)$ where $n_{V}(x)$ denotes the number of neighbors of $x$ in $V$. This gives $A_{1}$; now proceed in the same way with $A_{1}$, etc. until no further vertices can be removed. The output $A$ of the algorithm is the set of remaining vertices. Recurrence is then characterized by "burnability", i.e., $\eta \in \mathcal{R}_{n}$ if and only if $A=\emptyset$, i.e., all vertices can be burned.

The stationary measure $\lambda_{n}$ is thus the uniform probability measure on all burnable configurations in $V_{n}$. The cardinality $\left|\mathcal{R}_{V}\right|$ (= the number of recurrent configurations in $V$ ) equals the determinant of the 
discrete Laplacian on $V$ with open boundary conditions see 2]. E.g. if $V$ is a square containing $N$ sites, then $\left|\mathcal{R}_{V}\right| \simeq(3.21)^{N}$.

For a proof of these facts, see e.g. [2, 7], 9] or [12. Remark that for all finite $V \subset \boldsymbol{Z}^{2}$, the constant configurations $\eta \equiv 4$ and $\eta \equiv 3$ are in $\mathcal{R}_{V}$, but $\eta \equiv 2$ and $\eta \equiv 1$ are not recurrent except for some very special choices of $V$. One easily concludes that the condition that $\eta \in \mathcal{R}_{n}$ is a nonlocal hard-core constraint.

The following proposition is an immediate consequence of the burning algorithm.

Proposition 1.1. If $\eta \in \mathcal{R}_{n}$ and $\xi \geq \eta$ (pointwise), then $\xi \in \mathcal{R}_{n}$.

In some aspects the abelian sandpile measure $\lambda_{n}, n \rightarrow \infty$, behaves as a model of statistical mechanics at the critical point, a phenomenon which is sometimes referred to as "self-organized criticality" because there is no explicit tuning of parameters. In the physics literature various critical exponents related to the avalanche behavior are introduced for that model. One signature of "critical behavior" is the presence of power law decay of correlations for the height 1 two-point function, as proven by Majumdar and Dhar in [4]:

Proposition 1.2. There exist constants $c, C>0$ such that

$$
c|x|^{-4} \leq\left|\lambda_{n}(\eta(0)=\eta(x)=1)-\lambda_{n}(\eta(0)=1) \lambda_{n}(\eta(x)=1)\right| \leq C|x|^{-4}
$$


for all $x \neq 0$ and $n$ large enough.

On the other hand, a contour of 4's completely decouples the inside and the outside, as we now show. A subset $V \subset Z^{2}$ is called simply connected if the corresponding $\hat{V} \subset \mathbb{R}^{2}$ obtained by "filling the squares" of $V$ is simply connected.

Proposition 1.3. For $W \subset V_{n}$ denote by $4_{W}$ the event that $\eta(x)=4$ on $W$. For any simply connected subset $V \subset Z^{2}$ with $\bar{V} \subset V_{n}$ :

$$
\lambda_{n}\left(\eta_{V} \eta_{V_{n} \backslash \bar{V}} \mid 4_{\partial V}\right)=\lambda_{n}\left(\eta_{V} \mid 4_{\partial V}\right) \lambda_{n}\left(\eta_{V_{n} \backslash \bar{V}} \mid 4_{\partial V}\right)
$$

Proof. Denote by $\mathcal{R}_{V_{n} \backslash \bar{V}}^{\text {ext }}$ the set of configurations which are burnable in $V_{n} \backslash \bar{V}$ and such that the extension $\eta_{V_{n} \backslash \bar{V}} 4_{\bar{V}}$ is burnable in $V_{n}$. By the burning algorithm, $\eta_{V} 4_{\partial V} \eta_{V_{n} \backslash \bar{V}} \in \mathcal{R}_{V_{n}}$ if and only if $\eta_{V} \in \mathcal{R}_{V}$ and $\eta_{V_{n} \backslash \bar{V}} \in \mathcal{R}_{V_{n} \backslash \bar{V}}^{e x t}$. Therefore

$$
\begin{gathered}
\lambda_{n}\left(\eta_{V} \eta_{V_{n} \backslash \bar{V}} \mid 4_{\partial V}\right)=\frac{I\left[\eta_{V} \in \mathcal{R}_{V}, \eta_{V_{n} \backslash \bar{V}} \in \mathcal{R}_{V_{n} \backslash \bar{V}}^{e x t}\right]}{\left|\mathcal{R}_{V_{n} \backslash \bar{V}}^{e x t}\right|\left|\mathcal{R}_{V}\right|} \\
\lambda_{n}\left(\eta_{V} \mid 4_{\partial V}\right)=\frac{I\left[\eta_{V} \in \mathcal{R}_{V}\right]}{\left|\mathcal{R}_{V}\right|}
\end{gathered}
$$

and

$$
\lambda_{n}\left(\eta_{V_{n} \backslash \bar{V}} \mid 4_{\partial V}\right)=\frac{I\left[\eta_{V_{n} \backslash \bar{V}} \in \mathcal{R}_{V_{n} \backslash \bar{V}}^{e x t}\right]\left|\mathcal{R}_{V}\right|}{\left|\mathcal{R}_{V_{n} \backslash \bar{V}}^{e x t}\right|\left|\mathcal{R}_{V}\right|}
$$

which gives the result. 
1.3. Sandpile model with Potts interaction. Define the probability measures $\mu_{\beta, n}^{a}$ on $\Omega_{n}$ as

$$
\mu_{\beta, n}^{a}(\eta)=\frac{\exp \left(-\beta H_{n}(\eta \mid a)\right) I\left[\eta \in \mathcal{R}_{n}\right]}{\Xi_{\beta, n}^{a}}
$$

where the normalizing constant $\Xi_{\beta, n}^{a}$ is

$$
\Xi_{\beta, n}^{a} \equiv \sum_{\eta \in \mathcal{R}_{n}} \exp \left(-\beta H_{n}(\eta \mid a)\right)
$$

Similarly, we define the partition function $\Xi_{\beta, V}^{a}$ in an arbitrary finite volume $V$. This partition function will also be abbreviated as $\Xi_{a, V}$. $\mu_{\beta, n}^{a}$ is of course just the original Potts measure conditioned on being recurrent:

$$
\mu_{\beta, n}^{a}(\eta)=\nu_{\beta, n}^{a}\left(\eta \mid \mathcal{R}_{n}\right)
$$

Obviously, at infinite temperature, $\beta=0$, we recover the stationary measure $\lambda_{n}$ of the sandpile model. The constraint $\eta \in \mathcal{R}_{n}$ can be viewed as introducing an extra nonlocal hard-core interaction (implicitly given by the burning algorithm) but it also breaks the Pottssymmetry: approximately for $n \uparrow+\infty, \lambda_{n}(\eta(0)=4)=0.4, \lambda_{n}(\eta(0)=$ $3)=0.3, \lambda_{n}(\eta(0)=2)=0.2, \lambda_{n}(\eta(0)=1)=0.1$ as computed by Priezzhev, [10]. 


\section{Results}

With boundary condition $a=4$, at low temperature, the typical configurations of the Potts model on sand look like an ocean of 4's with exponentially damped burnable islands.

Theorem 2.1. For any $\epsilon>0$ there exists $\beta_{0} \in(0, \infty)$ such that for all $\beta>\beta_{0}$ and all $n \in \mathbb{N}$

$$
\mu_{\beta, n}^{4}(\eta(0)=4)>1-\epsilon
$$

Moreover there exists $c>0$ such that for $\beta>\beta_{0}$ and $n$ big enough we have the bound

$$
\left|\mu_{\beta, n}^{4}(\eta(x) \eta(0))-\mu_{\beta, n}^{4}(\eta(x)) \mu_{\beta, n}^{4}(\eta(0))\right| \leq e^{-c|x|}
$$

exponentially small in the distance $|x|$ from the origin.

(2.2) must be contrasted with the situation for $\beta=0$ where there are long range correlations, see Proposition 1.2

Besides "all 4", the "all 3" is the only other groundstate. But that one is unstable:

Theorem 2.2. For every $\alpha>0$ there exists $\beta(\alpha) \in(0, \infty)$ and $c=$ $c(\alpha, \beta)>0$ such that for all $\beta>\beta(\alpha)$

$$
\mu_{\beta, n}^{3}\left(\left|\left\{x \in V_{n}: \eta(x)=3\right\}\right|>\alpha\left|V_{n}\right|\right) \leq e^{-c\left|V_{n}\right|}
$$


for large $n$.

The next section gives the proof of Theorem 2.1 and introduces a random cluster representation of the Potts model on sand. Section 4 is devoted to the proof of Theorem 2.2. It will be seen that, as an extension of Theorem 2.2. for no matter what boundary conditions, the density of 4 tends to one with $\beta \uparrow+\infty$.

\section{RANDOM CLUSTER REPRESENTATION}

The volume $\overline{V_{n}}=V_{n} \cup \partial V_{n}$ can be considered as a finite graph with the sites $x \in V_{n} \cup \partial V_{n}$ as vertices and with edge set $B_{n}=B$ consisting of the nearest neighbor bonds $x \sim y$ where at least one neighbor is in $V_{n}$. We define the sand-Potts random cluster measure $\varphi_{p, n}^{a}=\varphi_{p}^{a}$ on this graph with parameter $p \in[0,1]$ as the probability measure on $\{0,1\}^{B}$ which to each $\sigma \in\{0,1\}^{B}$ assigns probability

$\varphi_{p}^{a}(\sigma)=\frac{1}{N_{p}^{a}}\left[\prod_{e \in B} p^{\sigma(e)}(1-p)^{1-\sigma(e)}\right] \sum_{\eta \in \mathcal{R}_{n}} I[\eta$ is constant on clusters of $\sigma]$

By cluster we mean a (nearest neighbor) connected component of sites (including isolated sites) as obtained from the bond configuration $\sigma$. Bonds for which $\sigma(e)=1, \sigma(e)=0$ are called open, respectively, closed. In this definition, we assume that the boundary sites are all connected (wired). All sites that are connected to the boundary are in the same 
cluster. The restriction that $\eta$ is constant on clusters also implies that $\eta$ is constant equal to $a$ on the cluster of the boundary. Remember however that $\eta \equiv 2$ and $\eta \equiv 1$ are not in $\mathcal{R}_{n}$.

\subsection{Stochastic domination.}

Lemma 3.1. Let $a=3$ or $a=4$. For every edge $e=\langle x y\rangle$ in $B$ and every $\sigma_{B \backslash e} \in\{0,1\}^{B \backslash\{e\}}$,

$$
\varphi_{p}^{a}\left(\sigma(e)=1 \mid \sigma_{B \backslash e}\right)=p
$$

if $x$ and $y$ are connected via open edges in $\sigma_{B \backslash e}$. If, on the other hand, $x$ and $y$ are not connected via open edges in $\sigma_{B \backslash e}$, then we still have

$$
p \geq \varphi_{p}^{a}\left(\sigma(e)=1 \mid \sigma_{B \backslash e}\right)
$$

and for $a=4$,

$$
\varphi_{p}^{4}\left(\sigma(e)=1 \mid \sigma_{B \backslash e}\right) \geq \frac{p}{7-6 p}
$$

Proof. Let $\sigma \in\{0,1\}^{B}$. We write

$$
\sum_{\eta \in \mathcal{R}_{n}} I[\eta \text { is constant on clusters }]=k(n, a ; \sigma)
$$

for the number of recurrent configurations that are constant on the $\sigma$-clusters and fixed equal to $a$ for each site that is $\sigma$-connected to $\partial V_{n}$. It equals $\left|R_{n}\right|$ when all edges in $\sigma$ are closed. Obviously, $k(n, a ; \sigma) \leq\left|R_{n}\right|$ and $k(n, a ; \sigma)$ is decreasing in $\sigma$ and is increasing in $a$. 
For $a=3$ or $4, k(n, a ; \sigma) \geq 1$. Continuing with either $a=3$ or $a=4$, we have

$$
\frac{\varphi_{p}^{a}\left(1_{e} \sigma_{B \backslash e}\right)}{\varphi_{p}^{a}\left(0_{e} \sigma_{B \backslash e}\right)}=\frac{p k\left(n, a ; 1_{e} \sigma_{B \backslash e}\right)}{(1-p) k\left(n, a, 0_{e} \sigma_{B \backslash e}\right)}
$$

and hence

$$
\varphi_{p}^{a}\left(\sigma(e)=1 \mid \sigma_{B \backslash e}\right)=\frac{1}{1+\frac{\varphi_{p}^{a}\left(0_{e} \sigma_{B \backslash e}\right)}{\varphi_{p}^{a}\left(1_{e} \sigma_{B \backslash e}\right)}}=\frac{p}{p+(1-p) \frac{k\left(n, a ; 0_{e} \sigma_{B \backslash e}\right)}{k\left(n, a ; 1_{e} \sigma_{B \backslash e}\right)}}
$$

Abbreviate $\sigma^{0, e}=0_{e} \sigma_{B \backslash e}$ and $\sigma^{1, e}=1_{e} \sigma_{B \backslash e}$; they are both equal to $\sigma_{B \backslash e}$ off $e$ and $\sigma^{0, e}(e)=0$ and $\sigma^{1, e}(e)=1$.

To prove the first statement (3.2): suppose $x, y$ are connected via open edges in $\sigma_{B \backslash e}$, then every configuration $\eta \in \mathcal{R}_{n}$ compatible with $\sigma$ has $\eta(x)=\eta(y)$, and hence $k(n, a ; \sigma)$ does not depend on $\sigma(e)$ in that case. However, if $x$ and $y$ are not connected via open edges in $\sigma_{B \backslash e}$, then we must investigate the effect of merging two clusters. By making $e$ open, we connect two clusters and we must estimate the new number of recurrent configurations that are constant on clusters in terms of the old. Since always

$$
k\left(n, a ; \sigma^{0, e}\right) \geq k\left(n, a ; \sigma^{1, e}\right)
$$

we obtain (3.3) from (3.7). For the last statement (3.4), we combine Proposition 1.1 with (3.7). Suppose that $\eta \in \mathcal{R}_{n}$ and is constant on 
clusters $C_{1}$ and $C_{2}$ taking there the values $a_{1}$ and $a_{2}$ respectively. The new configuration $\xi$ defined as

$$
\xi(x)=\eta(x), x \notin C_{1} \cup C_{2}, \quad \xi(x)=\max \left\{a_{1}, a_{2}\right\}, x \in C_{1} \cup C_{2}
$$

is still recurrent and is constant on $C_{1} \cup C_{2}$. Moreover, if say $C_{1}$ is the boundary cluster, then necessarily $a_{1}=4$ and hence also $\max \left\{a_{1}, a_{2}\right\}=$ 4 remains compatible with the boundary. (This does not work with the boundary condition $a=3$.) Simple counting shows that the map $\eta \rightarrow \xi$ is at most seven to one, or

$$
k\left(n, 4 ; \sigma^{0, e}\right) \leq 7 k\left(n, 4, \sigma^{1, e}\right)
$$

Combination of (3.9) and (3.7) gives (3.4).

Let $\psi_{q}$ be the Bernoulli product measure on $\{0,1\}^{B}$ with density $q=\psi_{q}\left(\sigma_{e}=1\right)$

Proposition 3.2. The random cluster measure $\varphi_{p}^{4}$ stochastically dominates $\psi_{q}$ with $q=p /(7-6 p)$, i.e., $\varphi_{p}^{4}(\sigma(e)=1, e \in E) \geq \psi_{q}(\sigma(e)=$ 1, $e \in E)$ for all edge sets $E$. For $a=3,4, \varphi_{p}^{a}$ is stochastically dominated by $\psi_{p}$. Finally, $\varphi_{p}^{a}$ always stochastically dominates $\varphi_{p^{\prime}}^{a}$ for $0 \leq$ $p^{\prime} \leq p \leq 1$ 
Proof. The first and second statement follows directly from Lemma 3.1 see e.g. Theorem 4.8 in [6]. The last statement follows from (3.7) and (3.8).

3.2. Coupling. The previous construction and arguments are analogous to and inspired by the Fortuin-Kasteleyn representation of the standard Potts model. To recover the $q$-state Potts model, one should simply replace $\mathcal{R}_{n}$ in (3.5) with $\Omega_{n}$. Our next step, making a coupling between the $\eta$ - and the $\sigma$-field, is the analogue of the SwendsenWang-Edwards-Sokal coupling, 13, 5]. For a general reference, see [6]. We make a coupling $\mathbb{P}_{p, n}^{a}=\mathbb{P}_{p}^{a}$ between the Potts model on sand and the sand-Potts random cluster measure. Let $\mathbb{P}_{p}^{a}$ be the probability measure on $\Omega_{n} \times\{0,1\}^{B}$ constructed as follows. Assign first to each site in $V_{n}$ a sandvalue according to the probability measure $\lambda_{n}$ and each site at the boundary $\partial V_{n}$ gets the value $a$. Independently, let each edge in $B$ take the value 0 or 1 with probabilities $1-p$ and $p$ respectively. Secondly, condition on the event that no two neighboring sites (including sites at the boundary) with different heights have an open edge connecting them. In a formula,

$$
\mathbb{P}_{p}^{a}(\eta, \sigma)=\frac{1}{M_{p}^{a}} I\left[\eta \in \mathcal{R}_{n}\right] \prod_{e=\langle x y\rangle \in B}\left[p^{\sigma(e)}(1-p)^{1-\sigma(e)} I\left[\left(\eta_{x}-\eta_{y}\right) \sigma(e)=0\right]\right]
$$


where in the last indicator function it is understood that $\eta(z)=a$ for $z \in \partial V_{n}$

Proposition 3.3. Suppose $\beta=-\ln (1-p)$. Then, $\mu_{\beta, n}^{a}$ is the marginal of $\mathbb{P}_{p}^{a}$ projected on $\Omega_{n}$ and $\varphi_{p}^{a}$ is the marginal of $\mathbb{P}_{p}^{a}$ projected on $\{0,1\}^{B}$.

Proof. The proof is by direct computation. For example, if we sum over the $\sigma$ we have

$$
\sum_{\sigma} \prod_{e \in B}\left[p^{\sigma(e)}(1-p)^{1-\sigma(e)} I[(\eta(x)-\eta(y)) \sigma(e)=0]\right]=(1-p)^{I[\eta(x) \neq \eta(y)]}
$$

which determines $1-p=\exp [-\beta]$.

\subsection{Proof of Theorem 2.1; stability of the 4-phase. We apply}

Proposition 3.3

$$
\begin{aligned}
& \mu_{\beta, n}^{4}(\eta(0)=4) \\
\geq & \mathbb{P}_{p}^{4}(\eta(0)=4 \mid 0 \text { is in the cluster of the boundary }) \times \\
& \varphi_{p}^{4}(0 \text { is in the cluster of the boundary }) \\
= & \varphi_{p}^{4}(0 \text { is in the cluster of the boundary })
\end{aligned}
$$

and now Proposition 3.2 to conclude that

$$
\mu_{\beta, n}^{4}(\eta(0)=4) \geq \psi_{p /(7-6 p)}(0 \text { is connected to the boundary })
$$


which goes to one, uniformly in $n$ as $\beta=-\ln (1-p)$ goes to $+\infty$. That shows (2.1). For the exponential decay of correlations, (2.2), observe that by the very same argument as above one shows that for large $\beta$, in $\mu_{\beta, n}^{4}$ there is percolation of 4's uniformly in $n$. For each $n$ the $\mu_{\beta, n}^{4}$-probability that there is a nearest-neighbor path of 4's connecting the origin with the boundary $\partial V_{n}$ is not smaller than the percolation probability in the Bernoulli bond process with occupation probability $(1-\exp [-\beta]) /(1+6 \exp [-\beta])$. Moreover, we can always consider a rectangle parallel to $V_{n}$ between the origin and site $x$ with one side proportional to $|x|$ and the other side equal to $n$. Again by the same domination argument, the $\mu_{\beta, n}^{4}$-probability that in that rectangle, there is percolation of 4's from one side of $V_{n}$ to the opposite side is not smaller than $1-\exp [-c|x|]$ with $c \uparrow+\infty$ as $\beta \uparrow+\infty$, uniformly in $n$. Therefore, denoting that event by 'crossing', we have that

$$
\mid \mu_{\beta, n}^{4}(\eta(x) \eta(0))-\mu_{\beta, n}^{4}(\eta(x) \eta(0) \mid \text { crossing }) \mid \leq \exp [-c|x|]
$$

That can be combined with Proposition 1.3, if the origin and the site $x$ are separated by a path of 4's, then they are independent:

$$
\mu_{\beta, n}^{4}(\eta(x) \eta(0) \mid \text { crossing })=\mu_{\beta, n}^{4}(\eta(x) \mid \text { crossing }) \mu_{\beta, n}^{4}(\eta(0) \mid \text { crossing })
$$


For each of the two factors we can use the previous argument to conclude the proof.

\section{Instability of THE 3-PHASE}

In this section we prove Theorem 2.2 .

The main idea is to consider the restricted ensemble defined below (following ideas from [3]). Let $V$ be a finite (large) volume and $\eta \in \Omega_{V}$ a configuration in the volume $V$. Define the contours of $\eta$ as connected components of edges separating the different values of $\eta$ in neighboring sites (we now consider these sites as centers of unit squares and the edges are the sides of these squares). We suppose that the configuration $\eta \in \mathcal{R}_{V}$ is burnable. The set of configurations that have contours with lengths not exceeding 12 not touching $\partial V$ is denoted by $\mathcal{R}_{V}^{12}$. That set of configurations defines a restricted ensemble. It is easy to see that we have the following alternative: either the configuration $\eta$ is a sea of 4 with islands made from contours with length $\leq 12$, or it is a sea of 3 with such islands:

$$
\mathcal{R}_{V}^{12}=\mathcal{R}_{V, 4}^{12} \cup \mathcal{R}_{V, 3}^{12}
$$

Notice that a sea of 2 or 1 is not possible by burnability.

We list all subconfigurations of energy $\leq 12$ in the sea of 4 and in the sea of 3. Of course, these islands have to be burnable. In the figure, 
empty cells are part of the sea.

\subsection{Islands in the sea of 4 .}

(1) Energy $=4$ :

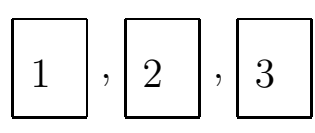

(2) Energy $=6$ :

\begin{tabular}{|l|l|}
\hline 2 & 2 \\
\hline
\end{tabular}, \begin{tabular}{|l|l|}
\hline 3 & 3 \\
\hline
\end{tabular} and rotations and reflections

(this remark will be omitted from here on)

(3) Energy $=7$

\begin{tabular}{|l|l|}
\hline 1 & 2 \\
\hline
\end{tabular}, \begin{tabular}{|l|l|}
\hline 1 & 3 \\
\hline
\end{tabular}

(4) Energy $=8$
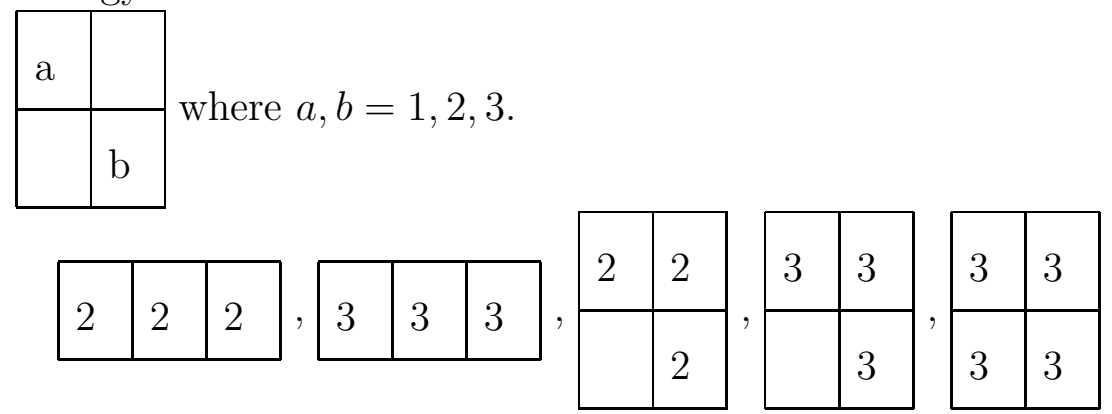

(5) Energy $=9$

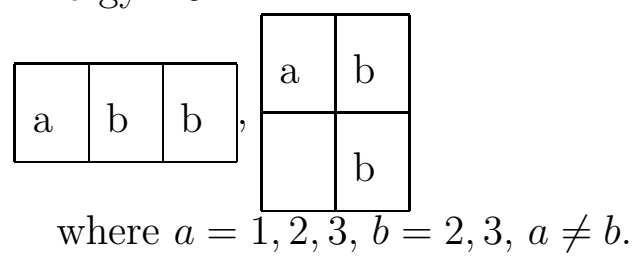


(6) Energy $=10$

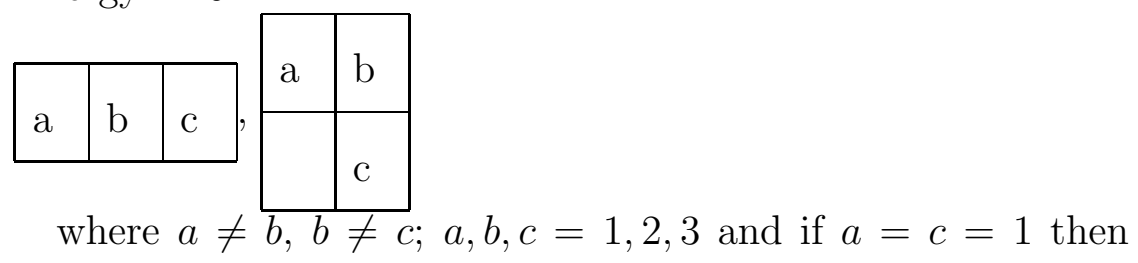

$b=3$

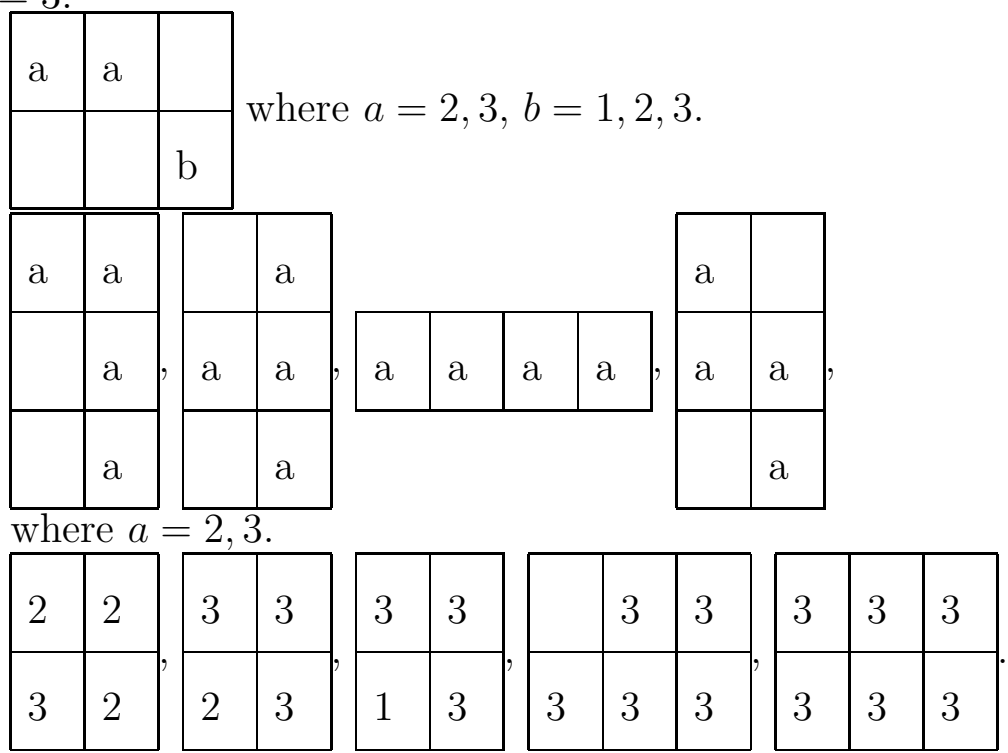

(7) Energy $=11$

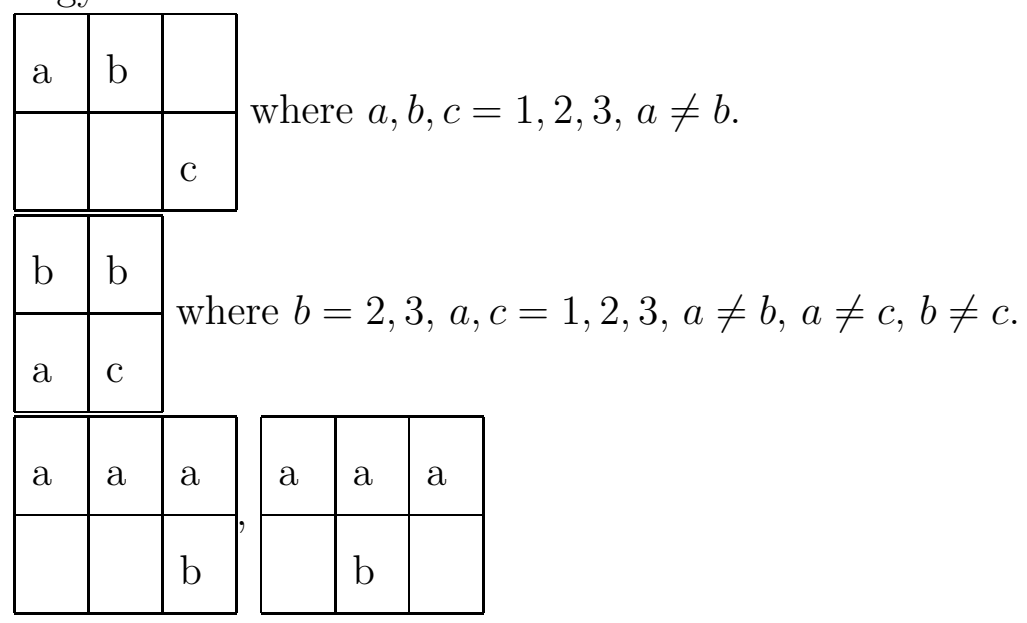




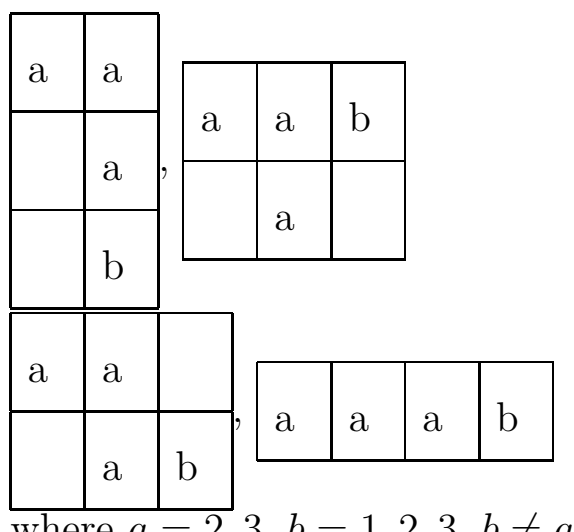

where $a=2,3, b=1,2,3, b \neq a$.

\begin{tabular}{|l|l|l|}
\hline 3 & 3 & \\
\hline 3 & 3 & $\mathrm{a}$ \\
\hline
\end{tabular}

(8) Energy $=12$

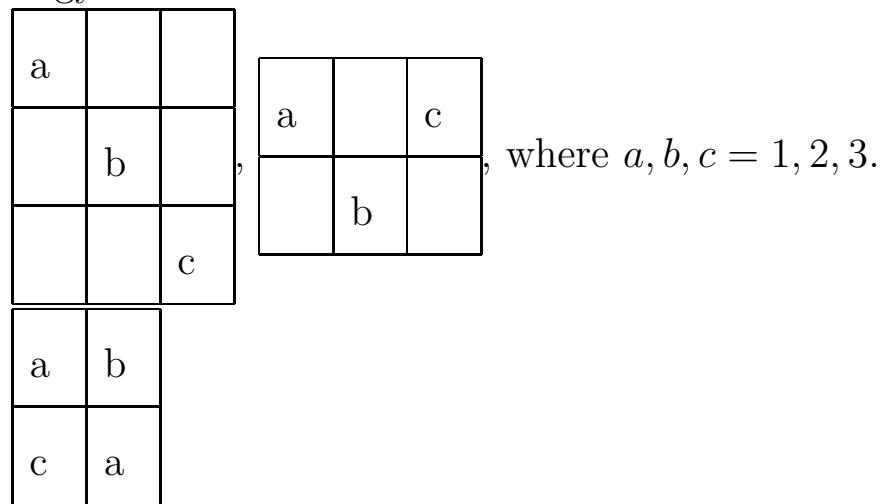

where $a, b, c=1,2,3, a \neq b, a \neq c$, if $a=1$ then $b=c=3$, if $a=2$, then $b=3$ or $c=3$.

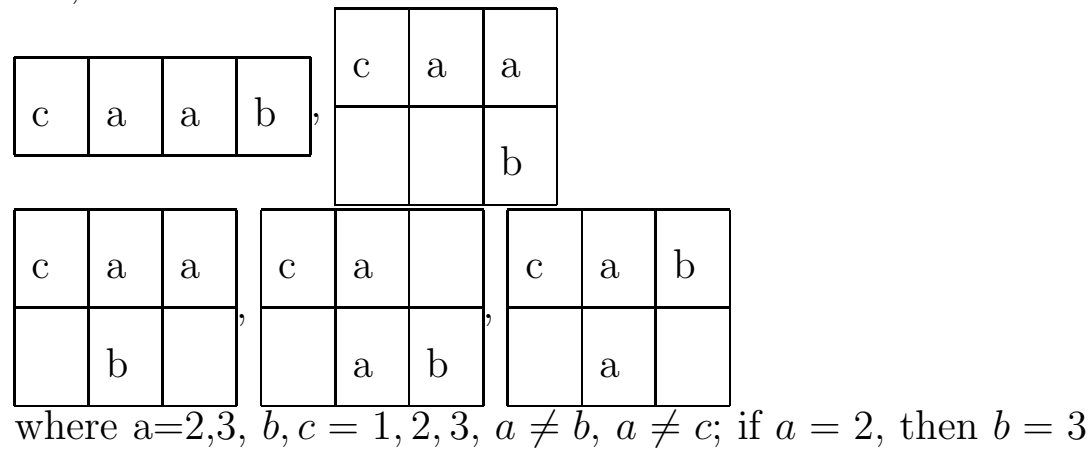

or $c=3$. 

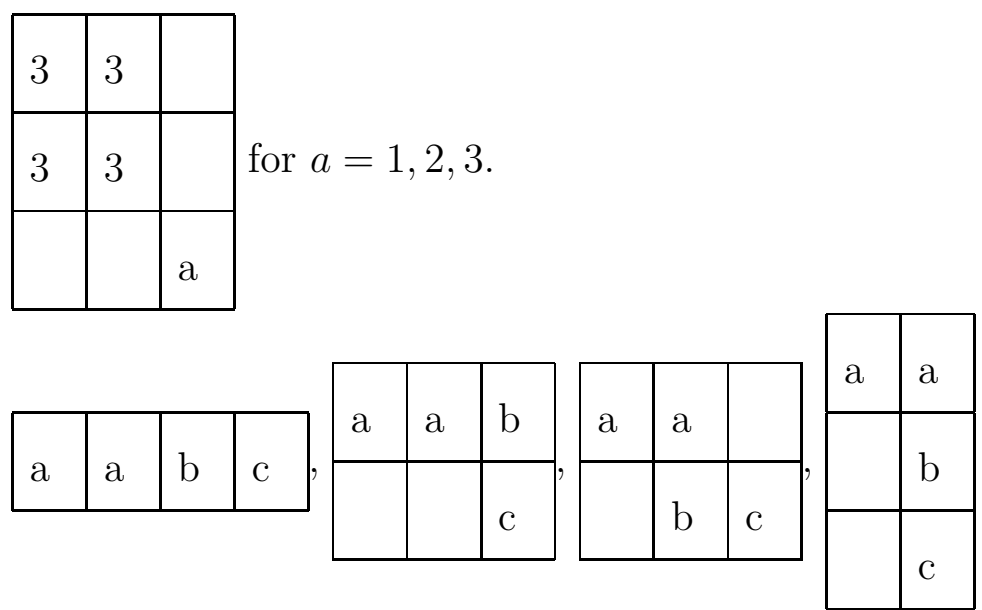

where $a, b, c=1,2,3, a \neq 1, a \neq b, b \neq c$.

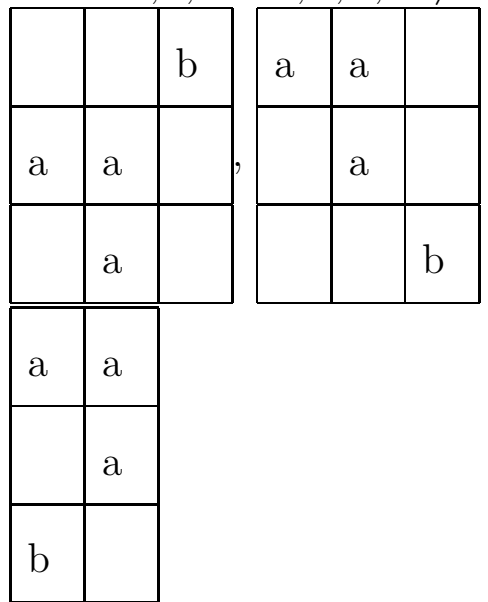

where $a=2,3$ and $b=1,2,3$.
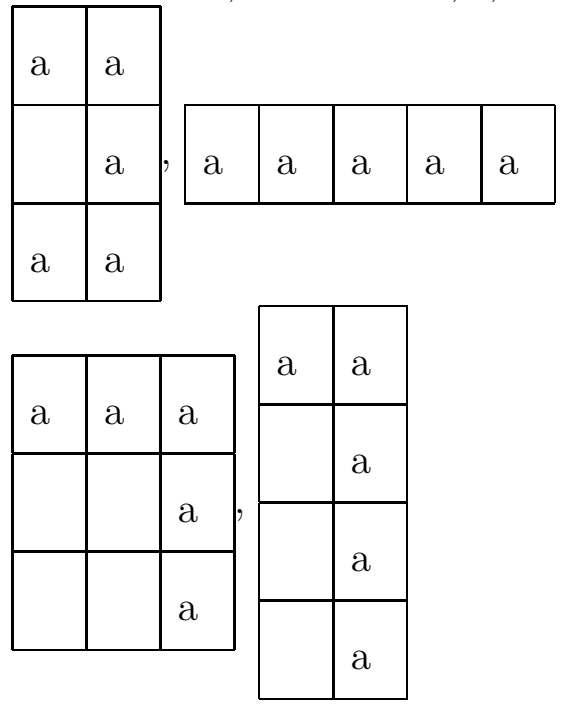


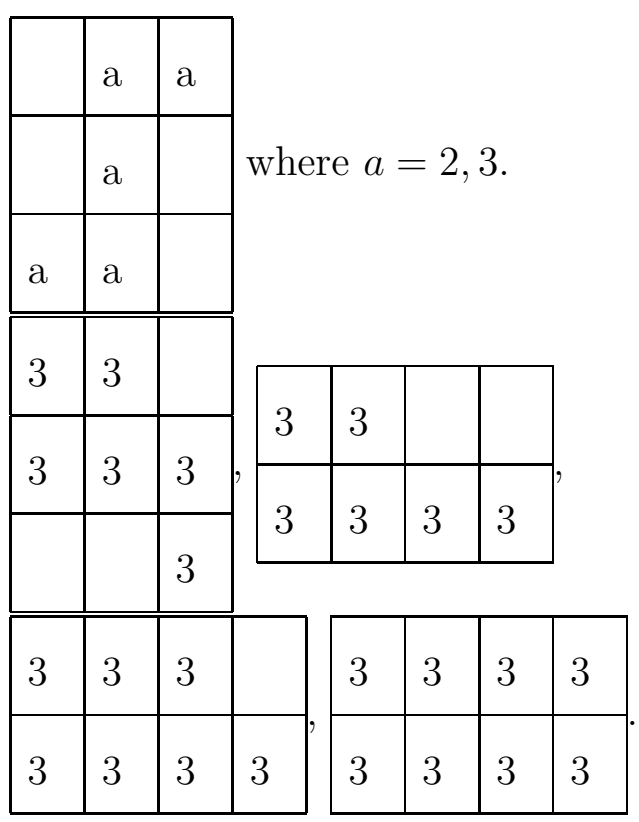

\subsection{Islands in the sea of 3.}

Lemma 4.1. Islands with energy $\leq 11$ in the sea of 3 are in oneto-one correspondence with islands with the same energy of 4. The correspondence is given by substitution $3 \longleftrightarrow 4$.

Lemma 4.2. The same correspondence can be applied to all islands with energy $=12$, with two exceptions:

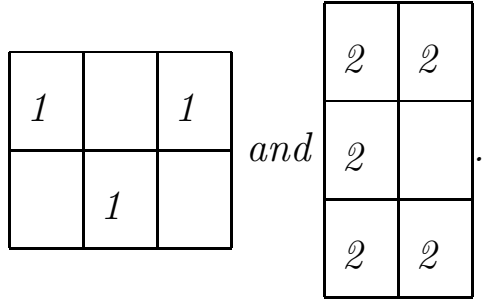

The correspondence gives all islands in the sea of 3 with energy 12.

Proof. This follows from the fact that 


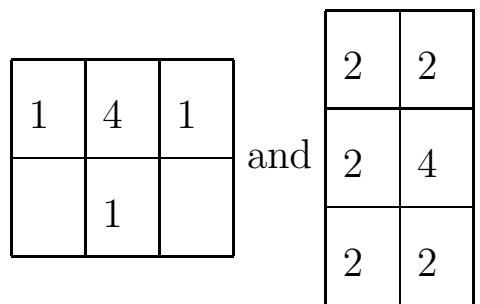

are burnable but

\begin{tabular}{|c|c|c|c|c|c|}
\hline \multirow{2}{*}{1} & & & \multirow{3}{*}{ and } & 2 & 2 \\
\hline & 3 & 1 & & & \\
\hline & \multirow{2}{*}{1} & & & 2 & 3 \\
\hline . & & & & 2 & \\
\hline
\end{tabular}

are not burnable.

4.3. Free energy estimates. Define $\Xi_{4, V}^{(12)}$ and $\Xi_{3, V}^{(12)}$ to be the partition functions of the ensembles $\mathcal{R}_{4, V}^{12}$, respectively $\mathcal{R}_{3, V}^{12}$.

Lemma 4.3. There exists $\beta_{0}$ such that for any $\beta>\beta_{0}$ there exists a constant $c>0$ such that for any sufficiently large volume $V$

$$
\log \Xi_{4, V}^{(12)}-\log \Xi_{3, V}^{(12)} \geq c|V| e^{-12 \beta}
$$

Proof. Define the extended ensemble $\mathcal{R}_{V, 3}^{12, \text { ext }}$, consisting of all configurations of disjoint burnable islands with energy $\leq 12$ in the sea of 3 in volume $V$. Evidently

$$
\mathcal{R}_{V, 3}^{12} \subset \mathcal{R}_{V, 3}^{12, e x t}
$$

and the inclusion is strict because e.g.

\begin{tabular}{|l|l|l|l|}
\hline 2 & 3 & 3 & 2 \\
\hline 2 & 3 & 3 & 2 \\
\hline
\end{tabular}


is a subconfiguration allowed in the ensemble $\mathcal{R}_{V, 3}^{12, \text { ext }}$ but not in the ensemble $\mathcal{R}_{V, 3}^{12}$. Indeed, it is not burnable but the island

\begin{tabular}{|l|l}
\hline 2 & is burnable. \\
\hline 2 &
\end{tabular}

If we analogously define $\mathcal{R}_{V, 4}^{12, \text { ext }}$, then in fact $\mathcal{R}_{V, 4}^{12, \text { ext }}=\mathcal{R}_{V, 4}^{12}$ because a configuration of (disjoint) islands in a sea of 4 is burnable if and only if all the individual islands are burnable in the sea of 4 , see Proposition 1.3

Therefore

$$
\Xi_{3, V}^{(12, e x t)} \geq \Xi_{3, V}^{(12)}
$$

and we can obtain Lemma 4.3 from the following

\section{Proposition 4.4.}

$$
\log \Xi_{4, V}^{(12)}-\log \Xi_{3, V}^{(12, e x t)} \geq c|V| e^{-12 \beta}
$$

The proof is an application of the usual polymer expansion (see [8. 11]) because islands in the sea of 4 do not interact (again from Proposition 1.3). Comparing the expansions of $\log \Xi_{4, V}^{(12)}$ and $\log \Xi_{3, V}^{(12, e x t)}$ term by term, and using Lemma 4.1 and Lemma 4.2, we obtain a difference in the terms of order $e^{-12 \beta}$ and no difference in previous terms. In the same way it is easy to prove a weaker inequality valid for all finite $V \subset \boldsymbol{Z}^{d}$ 
Proposition 4.5. For $\beta>\beta_{0}$ there exist $c>0, f \in \mathbb{R}$ such that for any finite $V \subset \boldsymbol{Z}^{d}$ :

$$
\log \Xi_{4, V}^{(12)}-\log \Xi_{3, V}^{(12)} \geq \log \Xi_{4, V}^{(12)}-\log \Xi_{3, V}^{(12, e x t)} \geq c|V| e^{-12 \beta}-f|\partial V|
$$

4.4. Proof of Theorem 2.2, Again, the connected components of the set of lattice edges separating different values of neighboring spins are called contours. Each contour $\gamma$ will be associated with values of spins on all squares touching $\gamma$. Consider the set $\Gamma=\left\{\gamma_{1}, \ldots, \gamma_{n}\right\}$ of all those contours of a given configuration $\eta \in \mathcal{R}_{V, 3}$ which have energy (length) exceeding $12,\left|\gamma_{i}\right|>12$. We call $\Gamma$ the polycontour of $\eta$. The polycontour separates the volume $V$ into a finite number of connected domains $V_{1}, \ldots, V_{m}$. To each domain $V_{i}$ is associated the value of the spin $\kappa_{i}$, induced by contours $\gamma_{j}$ neighboring this domain (so $\kappa_{i}=\kappa$ if the spins in the inner boundary of $V_{i}$ are equal to $\left.\kappa\right)$. If $\Gamma=\emptyset$ then there is the domain $V$, to which is associated $\kappa=3$. Denote the band consisting of squares touching $\Gamma$ by $[\Gamma]$. From burnability it follows that all domains $V_{i}$ for which $\kappa_{i}=1,2$ are contained in $[\Gamma]$. We denote by $V^{(3)}$ the union of those $V_{i}$ for which $\kappa_{i}=3$, and by $V^{(4)}$ the union of those $V_{i}$ for which $\kappa_{i}=4$. Now $\zeta$, the number of 3's in $V$, equals

$$
\zeta=\zeta^{(3)}+\zeta^{(4)}
$$


where $\zeta^{(3)}$, respectively $\zeta^{(4)}$ denotes the number of 3's in $V^{(3)}$, respectively $V^{(4)}$. Therefore

$$
\operatorname{Prob}[\zeta \geq \alpha|V|] \leq \operatorname{Prob}\left[\zeta^{(3)} \geq \frac{\alpha}{2}|V|\right]+\operatorname{Prob}\left[\zeta^{(4)} \geq \frac{\alpha}{2}|V|\right]
$$

We will separately estimate the two terms in the right-hand side. The last one is the easiest. By definition of $V^{(4)}$, all spin values equal to 3 in $V^{(4)}$ are contained in the islands listed above. Each island contains $\leq 8$ squares and has an energy $\geq 4$. So the energy per square is $\geq 1 / 2$. If $\zeta^{(4)} \geq|V| \alpha / 2$, then the energy of the configuration is $\geq \alpha|V| / 4$. Note that $\Xi_{3, V} \geq 1$ because the configuration $\equiv 3$ has zero energy. So the probability of the configuration $\eta$ is less than or equal $\exp (-\beta \alpha|V| / 4)$. As the total number of configurations does not exceed $4^{|V|}$,

$$
\mu_{\beta, V}^{3}\left(\zeta^{(4)} \geq \frac{\alpha}{2}|V|\right) \leq 4^{|V|} \exp \left(-\beta \frac{\alpha}{4}|V|\right)
$$

which, when $\alpha \beta>4 \log 4$, tends to zero as $|V| \rightarrow \infty$.

The rest of the proof is an estimate of $\mu_{\beta, V}^{3}\left(\zeta^{(3)} \geq \alpha|V| / 2\right)$.

Note that $\zeta^{(3)} \leq\left|V^{(3)}\right|$, and hence

$$
\mu_{V, \beta}^{3}\left(\zeta^{(3)} \geq \frac{\alpha}{2}|V|\right) \leq \mu_{V, \beta}^{3}\left(\left|V^{(3)}\right| \geq \frac{\alpha}{2}|V|\right)=\frac{Z_{\alpha}}{\Xi_{3, V}}
$$

where $Z_{\alpha}$ is the partition function of those configurations $\eta \in \mathcal{R}_{V, 3}$ for which $\left|V^{(3)}\right| \geq \frac{\alpha}{2}|V|$. 
It is easy to see that

$$
Z_{\alpha} \leq \sum_{\Gamma} e^{-\beta|\Gamma|} \Xi_{3, V^{(3)}}^{(12)} \Xi_{4, V^{(4)}}^{(12)}
$$

where the sum runs over those polycontours $\Gamma$, for which $\left|V^{(3)}\right| \geq \frac{\alpha}{2}|V|$ $\left(\Gamma=\emptyset\right.$, for which $V^{(3)}=V$ is included $)$.

Let us now estimate $\Xi_{3, V}$. Consider only those configurations $\eta \in$ $\mathcal{R}_{V, 3}$ which also belong to $\mathcal{R}_{V^{\prime}, 4}^{12}$, where $V^{\prime}$ is $V$ without squares touching $\partial V$. Then we have

$$
\Xi_{3, V} \geq e^{-3 \beta|\partial V|} \Xi_{4, V^{\prime}}^{(12)}
$$

From the cluster expansion (or by direct counting),

$$
\Xi_{4, V^{\prime}}^{(12)} \geq e^{-h|\partial V|} \Xi_{4, V}^{(12)}
$$

for some constant $h>0$. Therefore,

$$
\Xi_{3, V} \geq e^{-d|\partial V|} \Xi_{4, V}^{(12)}
$$

for some constant $d$ (depending on $\beta$ ). So we obtain

$$
\frac{Z_{\alpha}}{\Xi_{3, V}} \leq e^{d|\partial V|} \frac{\sum_{\Gamma} e^{-\beta|\Gamma|} \Xi_{3, V^{(3)}}^{(12)} \Xi_{4, V^{(4)}}^{(12)}}{\Xi_{4, V}^{(12)}}
$$

where the sum over $\Gamma$ is as in (4.7). In the right-hand side use that for every $\xi_{1} \in \mathcal{R}_{V^{(3)}, 4}^{12}$ and for every $\xi_{2} \in \mathcal{R}_{V^{(4)}, 4}^{12}$, there exists a $\zeta \in \mathcal{R}_{V, 4}^{12}$ that coincides with $\xi_{1}$ on $V^{(3)}$ and with $\xi_{2}$ on $V^{(4)}$. Hence,

$$
\Xi_{4, V}^{(12)} \geq \Xi_{4, V^{(3)}}^{(12)} \Xi_{4, V^{(4)}}^{(12)}
$$


and thus

$$
\frac{Z_{\alpha}}{\Xi_{3, V}} \leq e^{d|\partial V|} \sum_{\Gamma} e^{-\beta|\Gamma|} \frac{\Xi_{3, V^{(3)}}^{(12)}}{\Xi_{4, V^{(3)}}^{(12)}}
$$

Now use the inequality (4.4) in order to obtain the following estimate

$$
(4.6) \leq(4.13) \leq e^{d|\partial V|} \sum_{\Gamma} e^{-\beta|\Gamma|} e^{f\left|\partial V^{(3)}\right|} e^{-c\left|V^{(3)}\right| e^{-12 \beta}}
$$

But evidently $\left|\partial V^{(3)}\right| \leq|\partial V|+|\Gamma|$, and $V^{(3)} \geq \alpha|V| / 2$, so we have the estimate

$$
e^{(d+f)|\partial V|} e^{-\frac{1}{2} \alpha c|V| e^{-12 \beta}} \sum_{\Gamma} e^{-\beta^{\prime}|\Gamma|}
$$

where $\beta^{\prime}=\beta-f$. To estimate the last sum is standard combinatorics:

$$
\sum_{\Gamma} e^{-\beta^{\prime}|\Gamma|} \leq \prod_{x \in X}\left(1+\sum_{\gamma \ni x,|\gamma|>12} e^{-\beta^{\prime}|\gamma|}\right)
$$

where $X$ is the set of all vertices - ends of edges inside $V,|X| \leq|V|$. A classical Peierls' estimate gives

$$
\sum_{\gamma \ni x,|\gamma|>12} e^{-\beta^{\prime}|\gamma|} \leq H^{\prime} e^{-13 \beta^{\prime}} \leq H e^{-13 \beta}
$$

for some constants $H=H^{\prime} \exp [13 f]$. Since

$$
1+H e^{-13 \beta} \leq e^{H e^{-13 \beta}}
$$

we obtain the estimate

$$
(4.6) \leq e^{(d+f)|\partial V|} e^{-\frac{1}{2} \alpha c|V| e^{-12 \beta}} e^{H|V| e^{-13 \beta}}
$$


If $\beta>\log [2 H / \alpha c]$, this product tends to zero as $|V| \rightarrow \infty,|\partial V| /|V| \rightarrow$ 0. That together with (4.5) concludes the proof.

Remark 4.6. To prove that the density of 1's or 2's goes to zero is even easier: Let $N$ be the number of 1's and 2's in $V, N=N^{(3,4)}+N^{[\Gamma]}$ where $N^{(3,4)}$ is the number of 1's and 2's in $V^{(3)} \cup V^{(4)}$ and $N^{[\Gamma]}$ is the number of 1's and 2's in $[\Gamma]$. Observe that $N^{[\Gamma]} \leq 2|\Gamma|$, and the 1's and 2's in $V^{(3)} \cup V^{(4)}$ are contained in islands. For the islands, the energy

per square is $\geq 1 / 2$. The energy of a configuration is equal to $|\Gamma|$ plus the energy of the islands. Hence, if $N \geq \alpha|V|$, then that energy is not less than $\alpha|V| / 2$. We can then do as in (4.5) to prove that

$$
\mu_{\beta, V}^{3}(N \geq \alpha|V|) \rightarrow 0 \quad \text { as }|V| \rightarrow+\infty
$$

whenever $\alpha \beta>2 \log 4$.

The same proof as above remains valid for the ensemble $\mathcal{R}_{V, 4}$. In fact, it is valid modulo straightforward modifications, for general boundary conditions as well. The proof for the ensemble with general boundary conditions $\xi$ only needs small modifications concerning the islands touching the boundary.

\section{REFERENCES}

[1] Bak, P., Tang, K. and Wiesenfeld, K., Self-Organized Criticality, Phys. Rev. A 38, 364-374 (1988). 
[2] Dhar, D., The Abelian Sandpiles and Related Models, Physica A 263, 4-25 (1999).

[3] Dinaburg, E.L. and Sinai, Ya.G., An analysis of ANNNI model by Peierl's contour method, Commun. Math. Phys. 98, 119-144 (1985).

[4] Majumdar, S.N. and Dhar, D., Physica A 185, 129 (1992), J. Phys. A: Math. Gen. 24 L357 (1991).

[5] Edwards, R.G. and Sokal, A.D., Generalization of the Fortuin-KasteleynSwendsen-Wang representation and Monte Carlo algorithm Phys. Rev. D 38, 2009-2012 (1988).

[6] Georgii, H.-O., Häggström, O., Maes, C., The random geometry of equilibrium phases, Phase Transitions and Critical Phenomena, Vol. 18, Eds C. Domb and J.L. Lebowitz (Academic Press, London), 1-142 (2001).

[7] E.V. Ivashkevich, Priezzhev, V.B., Introduction to the sandpile model, Physica A 254, 97-116 (1998).

[8] M. Zahradnik, Analyticity of low-temperature phase diagrams of lattice spin models, J. Stat. Phys. 47, 725-755 (1987).

[9] Meester, R., Redig, F. and Znamenski, D., The abelian sandpile; a mathematical introduction, Markov Proc. Rel. Fields, 7, 509-523 (2002).

[10] Priezzhev, V.B., Structure of Two Dimensional Sandpile. I. Height Probabilities, J. Stat. Phys 93

[11] Seiler, E., Gauge Theories as a Problem of Constructive Quantum Field Theory and Statistical Mechanics, Lecture Notes in Physics 159. Springer, Berlin, 1982. 
[12] Speer, E., Asymmetric Abelian Sandpile Models, J. Stat. Phys. 71, 61-74 (1993).

[13] Swendsen, R.H. and Wang, J.-S., Nonuniversal critical dynamics in Monte Carlo simulations, Phys. Rev. Lett. 58. 86-88 (1987). 